\title{
XXXI. Cölestin von Scharfenberg in Sachsen.
}

Von

J. Stuber in Strassburg i. E.

(Mittheilungen aus dem mineral. Institut der Universität Strassburg, Nr. 15.)

(Mit 3 Figuren.)

Das hiesige mineralogische Institut erwarb im Winter dieses Jahres zwei Stufen von Cölestin aus den Erzlagerstätten von Scharfenberg bei Meissen.

Dieses Vorkommen ist schon längere Zeit bekannt, indem Werner, wie Breithaupt in Poggendorff's Annalen der Physik und Ghemie 1829, 11, 499 erwähnt, bereits zwei Exemplare, welche er für Schwerspath hielt, in seine Sammlung aufnahm. Breithaupt erkannte; dass diese von der Grube »Gute Goltes" und "König David " stammenden Krystalle Cölestin vom specifischen Gewichte 3,931 seien, und Frenzel giebt in seinem "mineralogischen Lexicon für das Königreich Sachsen " (S. 73) an, dass die Gombinationen $\bar{P} \infty \cdot \frac{1}{2} \bar{P} \infty . \infty \bar{P} \infty$ und $\breve{P} \infty \cdot \frac{1}{2} \bar{P} \infty . \bar{P}_{\frac{1}{2}}, \infty \bar{P} \infty$ vorkämen, sowie dass die Krystalle oft mehrfarbig, z. B. schön indigblau und ölgrün oder grünlich in der Mitte und an den Polen blau oder bläulich- und gelblichweiss etc. seien.

Die bergbaulichen Arbeiten in den letzten Jahren haben auf den Scharfenberger Erzlagerstätten mehrfach Drusen mit wasserhellen bis bläulichen Cölestinkrystallen geliefert. Doch wurden im Juli vorigen Jahres auch braune, offenbar durch fein beigemengtes Brauneisen gefärbte Krystalle aufgefunden, welche in ihrer Ausbildungsweise von den bläulichen Krystallen abweichen.

Das Vorkommen dieser letzteren Krystalle scheint ein sehr seltenes zu sein, da ausser einem im Juli vor. Jahres überfahrenen, eisenschüssigen Neste das ganze Ganggestein der über 100 Jahre alten Grube noch sonst nirgends Eisenverbindungen gezeigt haben soll. 
Es dürtte deshalb die Beschreibung der neuerdings aufgefundenen Cölestinkrystalle von Interesse sein.

Die schön himmelblauen, klar durchsichtigen, stark glänzenden Krystalle der einen Stufe erreichen eine Grösse von höchstens $7 \mathrm{~mm}$ in der Länge und $4 \mathrm{~mm}$ in der Dicke, bleiben aber meist noch unter diesen Dimensionen.

Bei einer Aufstellung, in welcher die Ebene der vollkommensten Spaltbarkeit als Basis und die beiden Ebenen, nach denen eine weitere Spaltbarkeit vorbanden ist, als primäres Prisma bezeichnet werden, also der Aufstellung, die schon von B reithaupt gewählt wurde und die auch den neueren Arbeiten ubber Gölestin zu Grunde liegt, zeigen die Krystalle ausser

Fig. 1.

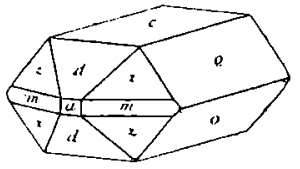
der von Frenzel erwähnten Fläche $\{011\} \breve{P} \infty$ (o in Fig. 1) auch die Basis $c=\{001\} 0 P$ gross und mit der ersteren im Gleichgewichte entwickelt. Dadurch erhalten die Krystalle, die meist senkrecht zur Brachydomenzone aufgewachsen sind, einen säulenförmigen Habitus. An dem freien Ende tritt ausser dem Makrodoma $d=\{102\} \frac{1}{2} \bar{P} \infty$ noch die von Frenzel nicht erwähnte primäre Pyramide $z=\{111\} P$, mit dem ersteren ebenfalls im Gleichgewichte, auf, so dass die Krystalle einer hexagonalen Säule mit Pyramide ähnlich sind. \{121\} $\mathscr{2} \breve{P} 2$ habe ich zwar an keinem der Krystalle nachweisen können, dagegen ist noch an manchen untergeordnet das primäre Prisma $m=\{110\} \infty P$ als kleine, glänzende Fläche vorhanden, und manchmal tritt noch, aber stets nur sehr klein, das Makropinakoid $a=\{100\} \infty \bar{P} \infty$ auf, welches parallel der c-Axe fein gestreift ist. Mit Ausnahme dieser letzten sind sämmtliche Flächen glatt und glänzend und geben sehr gute Reflexe.

Es wurden an diesen Krystallen folgende Winkel gemessen und berechnet :

\begin{tabular}{|c|c|c|}
\hline$(011):(01 \overline{1})=$ & $\begin{array}{l}\text { Gemessen: } \\
\text { *750 5 }{ }^{\prime}\end{array}$ & $\begin{array}{c}\text { Berechnet } \\
-\end{array}$ \\
\hline$(102):(10 \overline{2})=$ & $* 101 \quad 10$ & - \\
\hline$(111):(T 11)=$ & $90 \quad 17$ & $90^{0} 35^{\prime}$ \\
\hline$(111):(1 \overline{1} 1)=$ & $51 \quad 16$ & 5114 \\
\hline$(110):(1 T 0)=$ & $75 \quad 55$ & $75 \quad 58$ \\
\hline$(111):(102)=$ & $38 \quad 15$ & $38 \quad 15$ \\
\hline$(102):(011)=$ & 6126 & $61 \quad 39$ \\
\hline
\end{tabular}

Aus den sebr genau bestimmbaren Winkeln von $(011):(01 \overline{1})=75^{0} 51^{\prime}$

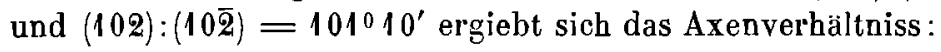

$$
a: b: c=0,78075: 1: 1,2834 .
$$

Die andere Stufe ist reichlich mit dunkelbraunen bis hellweingelben, durchscheinenden und in kleineren Individuen durchsichtigen Krystallen 
besetzt, welche in ihrer Grösse von $17 \mathrm{~mm}$ in der Länge und $15 \mathrm{~mm}$ in der Dicke bis zu mikroskopischen Dimensionen sehwanken. Die grösseren Individuen lassen meist einen sehr schönen zonaren Aufbau von dunkelbraunen und hellgelben, durchsichtigen Scbichten erkennen, und zwal sind die äusseren Schichten heller gefärbt als die inneren.

Der Habitus der Krystalle ist von dem der blaulichen verschieden.

Durch vorwiegende Entwickelung des Brachydomas $o=\{011\} \breve{P}_{\infty}$, dessen Flächen eine Streifung senkrecht zur Brachyaxe erkennen lassen, erhalten die Krystalle meist einen rhombisch-prismatischen Habilus (vergl. Fig. 2). Sie sind gewöhnlich ebenfalls senkrecht zur Brachydomenzone aufgewachsen, und am freien Ende tritt das Makrodoma $d=$ \{102\} $\frac{1}{2} \bar{P} \infty$ auf; seltener sind sie mit der Basis oder dem Brachypinakoid mit dem Gestein verbunden.

Das Makrodoma $\{102\} \frac{1}{2} \vec{P} \infty$ zeigt eine Streifung parallel der Makroaxe, offenbar durch Alterniren mit

Fig. 2.

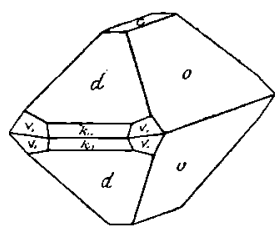
einem steileren Makrodoma $k_{1}$, welches die Combinationskante der Makrodomenflächen (102) und (10采) zuschärft. Dasselbe liess sich als $\{908\} \frac{9}{8} \bar{P} \infty$, ein für den Cölestin neues Makrodoma, bestimmen.

Diese Flächen sind meist sehr gerundet und gestreift und geben deshalb lang ausgezogene und nur sehr schwache Reflexe.

Ausserdem konnte noch durch annähernde Messung die für den Cölestin ebenfalls neue Pyramide $v_{1}=\{524\} \frac{5}{4} \bar{P} \frac{5}{2}$ nachgewiesen werden, welche die vierseitigen Ecken, die durch die Combination von $\{011\} \breve{P}_{\infty}$ und $\{908\}{ }_{8}{ }_{8} \bar{P} \infty$ entstehen, modificirt. Nur an den kleineren Krystallen ist diese Pyramide deutlicher entwickelt; an den grösseren äussert sie sich in Verbindung mit den noch zu besprechenden Flächen als eine starke Rundung der an der genannten Ecke zusammenstossenden Flächen.

Die Basis $c=\{001\} 0 P$ tritt gelegentlich, aber meist untergeordnet, seltener etwas grösser entwickelt, als stark glänzende Fläche hinzu, und manche Krystalle zeigen, aber nur sehr klein ausgebildet, die primäre Pyramide $z=\{111\} P$ glatt und sehr gut reflectirend (Fig. 3). Die Combinationskante der letzteren Fläche mit $\{524\} \frac{5}{4} \bar{P} \frac{5}{2}$, ebenso wie diejenige von $\{524\} \frac{5}{4} \bar{P} \frac{5}{2}$ mit $\{908\} \frac{8}{8} \bar{P} \infty$ ist stets stark gerundet.

Zu den bisher erwähnten Flächen treten noch, nur an einigen Krystallen in deutlicher Ausbildung nachweisbar, die folgenden hinzu, welche da, wo sie nicht in deutlicber Entwickelung vorhanden sind, sich

Fig. 3.

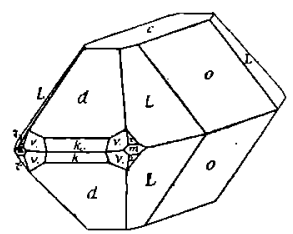
durch eine auffallende Streifung auf einigen Flächen oder Rundung einzelner Kanten bemerkbar machen, indem sie mit den bisher besprochenen Flächen alterniren. 
Besonders ist die Streifung auf fast allen Krystallfichen (ausgenommen sind nur die Flächen von $\{111\} P$ und $\{001\} 0 P)$ sehr auffallend und erleichtert die krystallographische Orientirung ausserordentlich.

In der Zone von $o=\{011\} \breve{P}_{\infty}$ zu $z=\{111\} P$ findet sich eine Pyramide der brachydiagonalen Reibe mit geringer Neigung gegen $o$, die sich als $L=\{155\} \breve{P} 5$ bestimmen liess (Fig. 3). Auch diese Form ist bisher vom Cölestin noch nicht bekannt.

Die Fläche ist besonders an den grösseren Individuen deutlich und ziemlich gross entwickelt und bewirkt ausserdem die an allen Krystallen zu beobachtende Streifung auf $\{011\} \breve{P}_{\infty}$ parallel der Combinationskante mit dieser Fläche.

Ist die Fläche nur klein ausgebildet, so bewirkt sie zusammen mit $\{111\} P$ eine Rundung der Combinationskante von $\{011\} \breve{P}_{\infty}$ und $\{524\} \frac{5}{4} \bar{P} \frac{5}{2}$. In der Zone von (111) zu (11T) findet sich selten und stets nur ausserordentlich klein eine Fläche, welche die Combinationskante der beiden primären Pyramiden gerade abstumpft, also gegen beide gleich geneigt ist ( $m$ in Fig. 3). Diese Fläche ist demnach als $\{110\} \infty P$ zu bezeichnen. Wenn sie auch nur sehr selten als gerade Abstumpfung an der Kante (111): (11T) zu beobachten ist, so ist sie doch fast immer in der auf $v_{1}=\left\{5_{24}\right\} \frac{5}{4} \bar{P}_{\frac{5}{2}}$ auftretenden Streifung zu erkennen, welche parallel der Combinationskante dieser Pyramide mit dem Prisma $\{110\} \infty P$ geht; wenn man die Zone von (111) zu (1/T) justirt hat, so erhält man meist in der Mitte zwischen den beiden Flächen einen deutlichen Reflex.

Mil dem Prisma alternirt derart, dass durch dieselbe eine grobe Streifung erzielt wird, die Pyramide $v_{1}=\left\{\boldsymbol{5 2}_{4}\right\}$. Auf der dem Makrodoma $k_{1}=$ $\{908\}$ zugewendeten Seite der Pyramide $v_{1}$ tritt zu der groben Streifung nach dem Prisma $m$ noch eine etwas zartere Riefung parallel der. Combinationskante mit $k_{1}$ hinzu. Auch auf dem Makrodoma $k_{1}=\{908\}$ ist nach der zuletzt genannten Combinationskante mit $v_{1}$, und zwar sowohl nach der rechts als nach der links gelegenen Kante, eine Streifung vorhanden, welche die ganze Fläche wie angeätzt erscheinen lässt.

Folgende Winkel wurden an den braumen Krystallen gemessen und berechnet:

\begin{tabular}{|c|c|}
\hline Gemessen : & Berechnet \\
\hline$(011):(001)={ }^{*} 52021^{\prime}$ & 一 \\
\hline$(102): ! 001)={ }^{*} 3936$ & - \\
\hline$(908):(90 \overline{8})=56 \cdot 26$ & $56^{0} 29^{\prime}$ \\
\hline$(011):(102)=6151$ & 6155 \\
\hline$(102):(111)=3821$ & $38 \quad 22$ \\
\hline$(111):(011)=4517$ & $45 \quad 18$ \\
\hline$(155):(011)=1128$ & 1126 \\
\hline$(111):(110)=2525$ & 2526 \\
\hline
\end{tabular}




$$
\begin{array}{rrr} 
& \text { Gemessen : } & \text { Berechnet: } \\
\left.(102):(524)=29^{0} 7^{\prime} \dagger\right) & 28^{0} 56^{\prime} \\
(011):(524)=6255 \dagger) & 6154 \\
(524):(52 \overline{4})=4834 \dagger) & 4932
\end{array}
$$

Die mit * bezeichneten genauer messbaren Winkelwerthe dienten zur Berechnung des Axenverhältnisses:

$$
a: b: c=0,78341: 1: 1,29618 \text {, }
$$

das von dem für die blauen Krystalle geltenden ziemlich beträchtlich abweicht.

Von den beiden Vorkommen wurde auf Spaltungsblättchen parallel der Basis der scheinbare stumpfe. Winkel der optischen Axen in $\alpha$-Monobromnaphtalin gemessen. Für die bläulichen Krystalle betrug derselbe

für die braunen

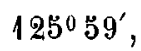

$$
124.050^{\prime} \text {. }
$$

Der erstere Winkel weicht um 10, der letztere um $2^{0}$ von dem von Liweh (diese Zeitschr. 12, 442) mitgetheilten Werthe (126055') ab.

Die optische Axenebene ist in beiden Fällen $\{010\} \infty \breve{P}_{\infty}$.

Line qualitative Analyse des braunen Vorkommens ergab einen kleinen Gehalt an $\mathrm{Fe}_{2} \mathrm{O}_{3}$. Dasselbe wurde auch spectralanalytisch auf $\mathrm{BaO}$ untersucht, ein Gehalt an letzterer war jedoch nicht nachweisbar.

f) Schimmermessung. 